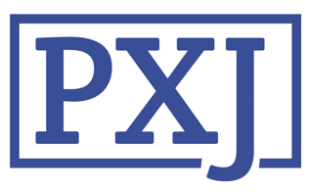

Patient Experience Journal

Volume 6 | Issue 3

Article 16

2019

\title{
Seven steps to successful change: How a large academic medical center prepared patients for organizational change
}

\author{
Brian Carlson \\ Vanderbilt University Medical Center \\ Madison Agee \\ Vanderbilt University Medical Center \\ Terrell Smith \\ Vanderbilt University Medical Center \\ Paul Sternberg Jr \\ Vanderbilt University Medical Center \\ Jason Morgan \\ Vanderbilt University Medical Center
}

Follow this and additional works at: https://pxjournal.org/journal

Part of the Health and Medical Administration Commons, Health Information Technology Commons, Health Policy Commons, Health Services Administration Commons, Health Services Research Commons, Nursing Commons, Organization Development Commons, Technology and Innovation Commons, and the Training and Development Commons

\section{Recommended Citation}

Carlson B, Agee M, Smith T, Sternberg P, Morgan J. Seven steps to successful change: How a large academic medical center prepared patients for organizational change. Patient Experience Journal. 2019; 6(3):129-138. doi: 10.35680/2372-0247.1385. 
Seven steps to successful change: How a large academic medical center prepared patients for organizational change

\section{Cover Page Footnote}

This article is associated with the Culture \& Leadership lens of The Beryl Institute Experience Framework. (http://bit.ly/ExperienceFramework). You can access other resources related to this lens including additional PXJ articles here: http://bit.ly/PX_CultureLeadership 


\title{
Seven steps to successful change: How a large academic medical center prepared patients for organizational change \\ Brian Carlson, Vanderbilt University Medical Center, b.carlson@vumc.org \\ Madison Agee, Vanderbilt University Medical Center, madison.m.agee@vumc.org \\ Terrell Smith, Vanderbilt University Medical Center, terrell.smith@vumc.org \\ Paul Sternberg, Jr., Vanderbilt University Medical Center, paul.sternberg@vumc.org \\ Jason Morgan, Vanderbilt University Medical Center, Jason@mjmorganconsulting.com
}

\begin{abstract}
Vanderbilt University Medical Center (VUMC) launched a new electronic health record (EHR) in a "big bang" implementation that saw the new software go live across multiple hospitals, clinics and geographic locations in a single morning. The organization rightly focused most of its energy on preparing its nearly 25,000 employees for the impacts of the transition, but it also considered the effects that would be felt by its patients and families. Survey data indicate that patient satisfaction scores demonstrably dip before, during and after an EHR implementation, and take approximately a year to recover. A team at DMC employed a seven-step approach to preparing patients for the impacts of the transition, which led to a return to pre-implementation patient satisfaction scores in about half the time of its peer institutions. The article explores these seven steps in detail and offers recommendations for how healthcare organizations facing largescale change can use a similar structured approach to mitigate negative impacts to patients.
\end{abstract}

\section{Keywords}

Patient experience, EHR, change management, communication, transformation, workforce preparation, patient satisfaction, project management, consumer experience

\section{Introduction}

Attend any event where healthcare industry leaders are in attendance, and from both the content of formal presentations and the subject of overheard conversations, it becomes immediately apparent that the clear majority of their organizations are facing at least one large-scale organizational change. Listen a little longer, and you might learn that the organizations not currently grappling with a big change likely have one just around the corner. A recent survey of healthcare CEOs revealed that $83 \%$ of them would describe the pace of innovation in healthcare as "speeding up" over the past year. The remaining 17\% characterized it as "staying the same," and not a single CEO indicated the pace of innovation was "slowing down". 1

When managing their organizations through changes such as mergers and acquisitions, alterations in executive leadership, organizational restructuring, or implementations of major systems such as an electronic health record (EHR), it is likely that healthcare leaders are often hyper-focused on the impact to their workforces (who usually bear the brunt of the change).2,3,4,5,6 Yet, they would be well-served to also consider the downstream effects on their patients, families and visitors. While a few older studies exist that stress the patient and family experience during transformational change, ${ }^{7,8}$ there does seem to be a gap in recent literature focusing on this issue.

\section{Background}

Consider the experience of Vanderbilt University Medical Center (VUMC), which implemented a new EHR (Epic) in the fall of 2017. VUMC's Epic implementation was truly a transformational change for the institution, involving not just clinical care areas (including inpatient and outpatient), but also key functions such as patient registration and scheduling, benefits coordination, and billing.

VUMC is one of the largest academic medical centers in the Southeast and is the primary resource for specialty and primary care in hundreds of adult and pediatric specialties for patients throughout Tennessee and the Mid-South. The medical center is the region's locus of postgraduate medical education, with over 1,000 residents and fellows training in more than 100 specialty areas. Vanderbilt University Adult Hospital and the Monroe Carell Jr. Children's Hospital at Vanderbilt are recognized each year by U.S. News \& World Report's Best Hospitals rankings as national leaders, with 19 nationally ranked adult and pediatric specialties. 
VUMC's size made the project already enormous, given its nearly 25,000 employees and volunteers, more than two million outpatient visits per year, 65,000 discharges from its three hospitals, and 100,000 emergency room visits. The project scope was made even more complex by the implementation schedule: going live with a "big bang" (all components at one time) rather than sequential implementation or "easing into it" with pilot areas.

As expected, VUMC focused much effort on supporting its employees through the switch to Epic, but the organization was also incredibly concerned with how the implementation would affect the experience of VUMC's patients. Existing literature linking EHR adoption and patient experience is not lacking; it appears, however, to focus more on the longer-term outcomes brought by a transition to a new EHR and less on the more immediate impact of an overall transformational change. $9,10,11,12$

A study by Press Ganey provided insight into how the implementation might affect patient experience in the specific time period of the Epic implementation. The study, which looked at the effect of an EHR implementation on patient experience scores, demonstrated a marked decrease in patient experience scores one quarter before and one quarter after an implementation. It also revealed that VUMC should expect it to take four quarters before patient experience scores returned to pre-go live levels (Figure 1). ${ }^{13}$ The Press Ganey study prepared VUMC leadership for the reality of downward pressure on VUMC's patient experience scores before, during and after the switch to Epic.
VUMC leadership formed a workgroup dedicated to preparing patients and families for the impacts of the Epic implementation. The workgroup's goal was to see VUMC return to pre-go live levels more quickly than the other 26 organizations in the Press Ganey study, which showed that it took at least a year to recover from the disruption in patient experience score performance.

\section{Preparing for Change}

This patient preparation workgroup was a multidisciplinary team comprising operational leaders, physicians, staff members, Epic experts, and most importantly, patient and family volunteers. Specifically, the workgroup was tasked with:

1. Identifying patient-visible variations created by the Epic implementation

2. Developing and executing on appropriate patient communication plans, including messaging, timing and communication methods/channels

3. Educating VUMC's workforce on how to communicate the change to patients, families and visitors

Given the lack of literature that focuses on considering the patient experience during transformational change, the workgroup did not have a research-driven blueprint for success in its approach. It instead relied on a combination of hypotheses, insights from strategies used in prior change management efforts, and best practices from other industry sectors to create seven distinct steps to achieve success, as outlined below.

\section{Figure 1. Patient Experience Scores - Pre/Post EMR Implementation (26 Systems Benchmarked)}

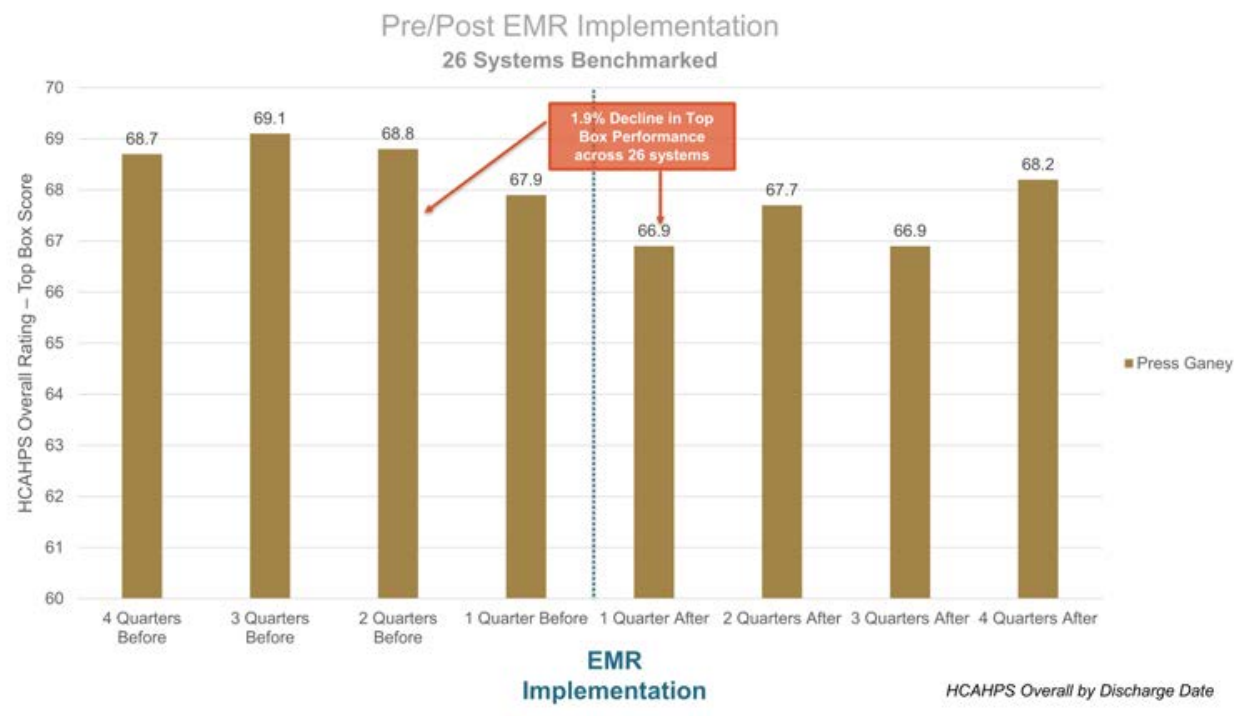




\section{Step 1: Include patients in the planning}

Customer-focused organizations facing a large-scale change would be well-advised to ask their patients how they think the change will affect their experience with the organization. ${ }^{14,15}$ Patients bring a valuable perspective to the table, often raising questions that may not be on the radar of stakeholders close to the change or eliminating concerns on which stakeholders had been placing unmerited value.

VUMC's patient preparation workgroup realized this early in the process, adding patient and family member volunteers as formal members of the team. These team members contributed significant feedback and insights throughout the life of the implementation effort, ensuring the inclusion of the patient perspective at every juncture.

The workgroup also collaborated extensively with VUMC's patient and family advisory councils, whose members were willing to donate both their time and thought leadership to the effort. The workgroup regularly presented on their efforts to the councils, soliciting their input for important decisions and seeking assurance that the workgroup's efforts were on the right trajectory.

To maximize the contributions of both the workgroup's permanent members and the ongoing feedback from the advisory councils, the workgroup provided important context for the EHR implementation project and its expected impacts. The patient and family perspective was also critical in identifying how the new EHR would be beneficial for patients and families, as well as strategizing how to minimize these effects and make the switch to Epic more seamless for VUMC's patients and families.

\section{Step 2: Align with governance}

Given the size and scope of the Epic implementation, VUMC created a formal governance structure that established, organized and oversaw multiple workgroups, including patient preparation. Throughout the project, the patient preparation workgroup worked closely with the governance structure, soliciting feedback on proposed plans, asking for support in addressing challenges, and lining up necessary resources for execution.

This formal governance structure was an essential element to the project's overall success, ensuring organizational alignment on the priorities and direction of the Epic implementation and providing a holistic view of the various interconnected elements such a massive organizational change entails (see Figure 2). Without such oversight, there is the potential to leave gaps in the decision-making process and to allow for confusion on "who's on first and who's on second". ${ }^{16}$

\section{Step 3: Identify patient-visible variations}

The workgroup also identified patient-visible variations, defined as any difference a patient or family member might experience at VUMC post-Epic go live. As part of this analysis, the group garnered input from Epic experts, VUMC employees, and other hospitals and health systems that had implemented Epic. The result was a list of dozens of variations, ranging from subtle to substantial.

Deciding how to manage this list and effectively communicate these impacts to VUMC's patients and families was a daunting proposition. This was where the input of the patient and family members became

\section{Figure 2. Organizational Oversight at VUMC}

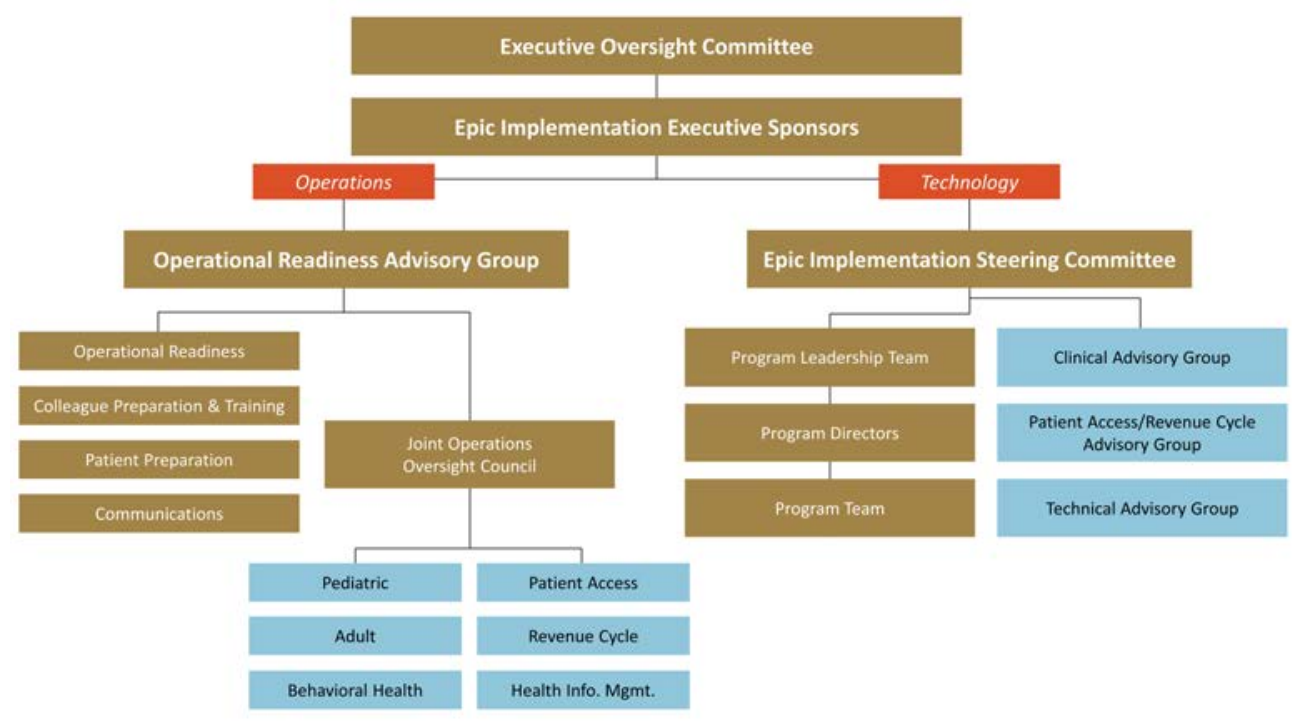


Figure 3. Patient Visible Variations

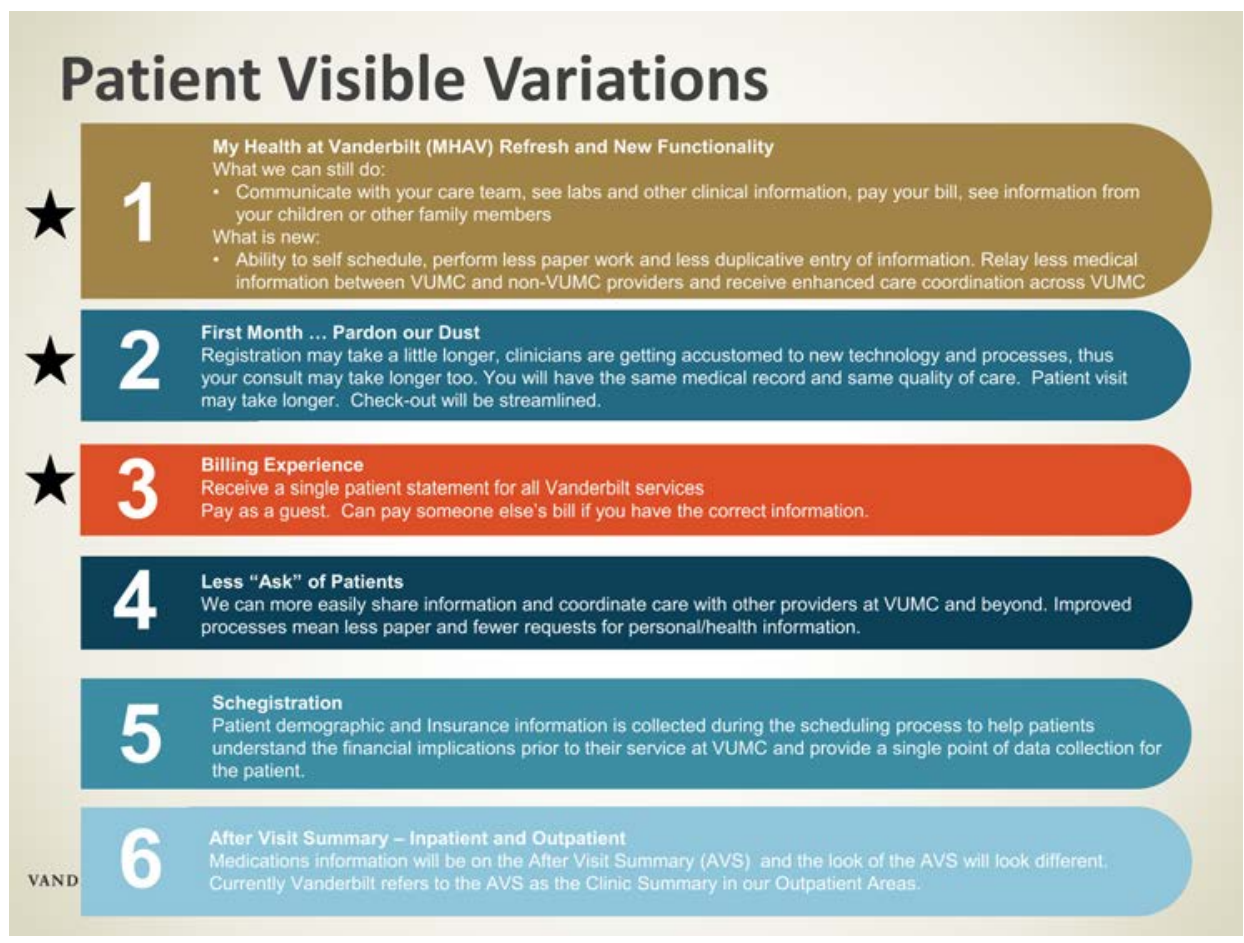

invaluable, as they offered two very important pieces of advice:

1. "Only tell us what we need to do, when we need to do it. Do not overwhelm us with a bunch of confusing messaging."

2. "This change is important and good, but it is your issue. Do not make it ours. We want to be the most important focus of attention."

Using this guidance, the workgroup distilled the original list down to a much more manageable list of six variations, of which three were considered the most important and merited the most energy from the workgroup: overall awareness of the transition, a better patient billing experience, and changes to the online patient portal (Figure 3).

\section{Step 4: Inform and prepare employees}

Because face-to-face (or telephone-based) interactions are the standard in healthcare situations, all the thoughtful work executed on by the team could be placed in serious jeopardy if VUMC colleagues were uncertain about, unprepared for, or unwilling to play their role in communicating the change to patients and families. Therefore, colleague education and preparation became the primary focus of the workgroup's efforts as the implementation date neared. ${ }^{17}, 18$
One difficulty in this effort was that there was no single, monolithic attitude among VUMC employees; many were excited about the switch to Epic, while others were decidedly more pessimistic about the change. Another complicating factor for workforce education was stressVUMC employees had been tasked with not only learning new software, but also adjusting to related changes in workflows that were being updated as part of the implementation. Because of VUMC's "big bang" switch, they had to put their recently learned skills into practice essentially overnight. This stress could cause even the employees who were models of customer service to act in unpredictable ways.

This variability and unpredictability meant that the workgroup needed to take an innovative approach to preparing employees how to communicate with patients regarding the change. Overall, the team incorporated helpful guidance, reinforcement of VUMC values, and humor into their plan.

Guidance was preferred over more prescriptive scripting, as feedback from other projects helped the team to understand that VUMC employees preferred the freedom to use language that felt natural to them in the moment. To help formulate this guidance, the workgroup once again engaged the patient and family advisory council, reviewing suggested language for various scenarios. These verbal messages culminated in a messaging matrix that was 
Figure 4. Communicating with Patients at Go Live

\section{Communicating with Patients at eStar Go Live}

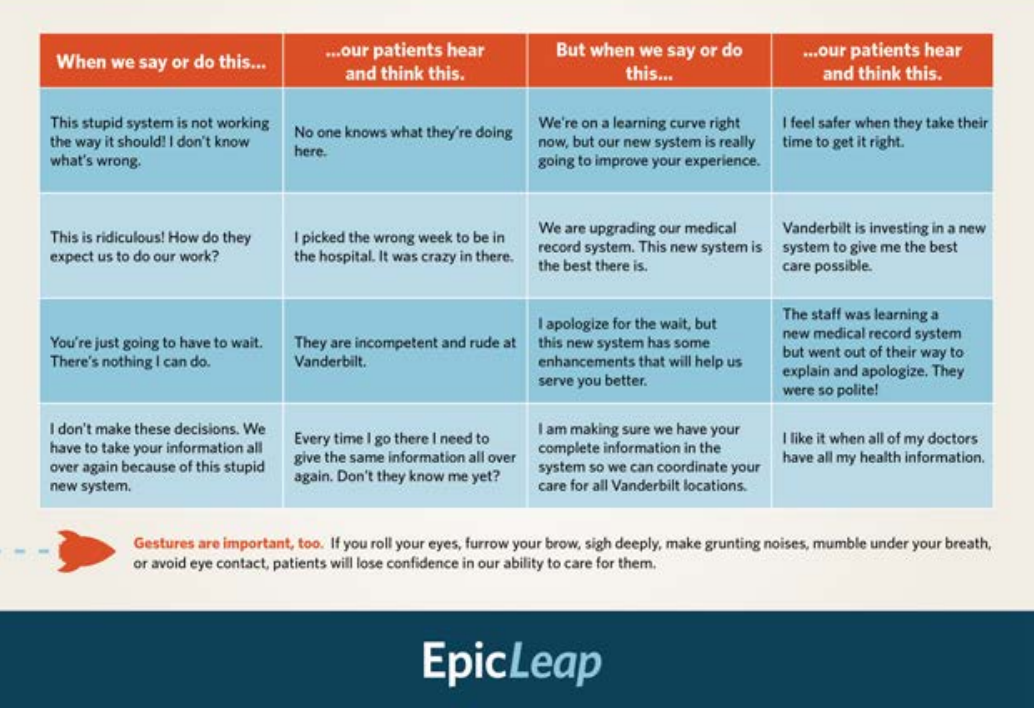

shared with staff in the days and weeks leading up to the go live (Figure 4).

The messaging matrix provided solid examples of positive and negative ways to guide conversations during the go live period. It also reminded employees that gestures and non-verbal communication were equally as important as the words they used during the transition.

The workgroup recognized that there were going to be hundreds of smaller changes specific to local areas that could either not be anticipated or, given resource availability, modeled in advance. To assist with these scenarios, the team provided an overarching framework that allowed local experts to develop a message unique and customized to their area (Figure 5).

Reinforcement of VUMC's values was also a central element in preparing the workforce. The team integrated key elements including VUMC's Credo, as well as its Patient and Family Promise, into the education it provided to employees. Part of this reinforcement was an ongoing reminder for employees to call on their professional selfreflection during the transition. Of course, they were going to feel significant stress because of the change, but it was important for them to remember that the patients in front of them would be more concerned about their own

Figure 5. Local Communication Recommendations

\section{Local Communication Recommendation}

$\begin{gathered}\text { What is the } \\ \text { change }\end{gathered} \quad \begin{gathered}\text { Why the } \\ \text { change }\end{gathered}-\begin{gathered}\text { What the change } \\ \text { means to the patient } \\ \text { (optional - if can describe) }\end{gathered}$

\section{Example:}

"You will notice we have new identification bands because we have upgraded our computer system."

If area feels patients need to be notified, do so verbally, not via signage. 
healthcare than whether a new EHR was elevating the anxiety of the staff. One council member remarked, "The change is your problem, not mine. Do not make it mine by making me feel like my problem is less important that yours".

To bring together the suggested messaging and values reinforcement, the workgroup created a video. ${ }^{19}$ As with the messaging matrix, the video, which featured actual VUMC employees, highlighted both "right" and "wrong" ways to communicate with patients about the transition to Epic. The "wrong" ways were purposefully depicted as slightly over-the-top, which injected a little levity and humor into a serious and stressful situation and helped employees better recall the information. This video was shown before every Epic training session (which were required of all employees using Epic), as well as during employee events and departmental meetings.

\section{Step 5: Inform patients}

The workgroup also needed to determine messaging, timing and channels for informing patients of these variations. ${ }^{20}$ It created a holistic campaign that created justin-time awareness for patients and families that VUMC was undergoing a technology change, electing to not create uncertainty or fear by communicating the change too far in advance.

Originally conceived as a "pardon our dust" campaign, which had been the core messaging used by several other

\section{Figure 6. VUMC Signage}

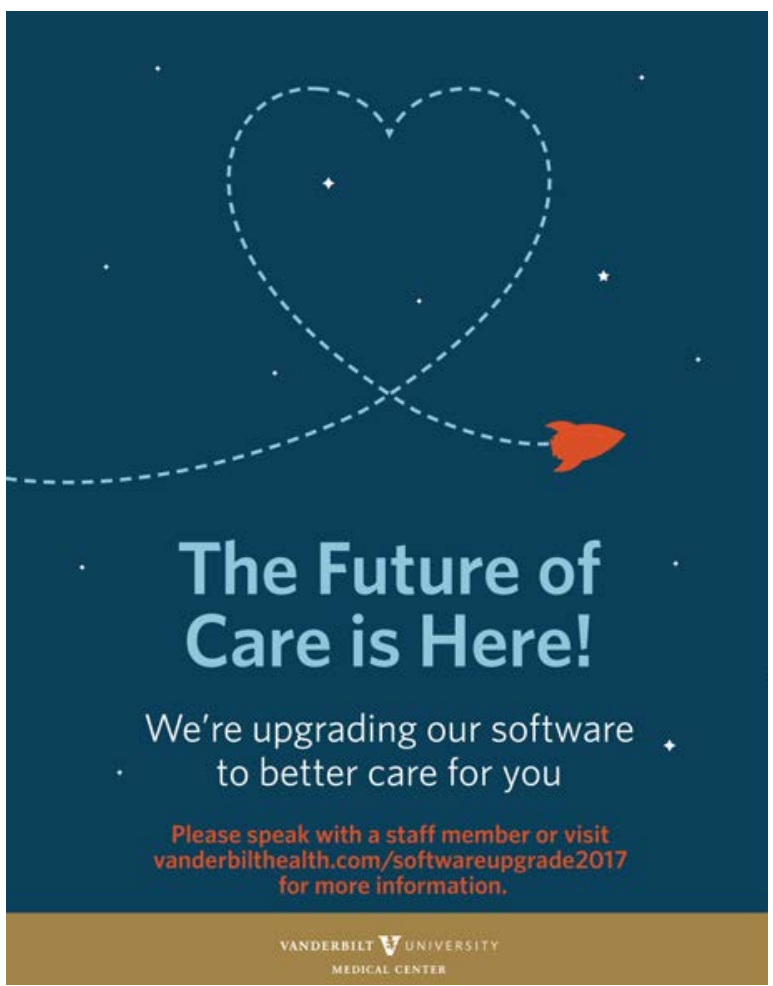

new EHR implementation sites, VUMC instead elected to use more aspirational, optimistic language. Rather than apologetically requesting patience, the "The Future of Care is Here!" campaign generated excitement for patients and families (Figure 6).

Deployed with signage throughout the medical center, the campaign provided context to patients and families who were receiving care during and after the switch. It also had the added bonus of buying VUMC some understanding from its patients that the organization was experiencing change.

The signage directed patients and families to learn more by visiting a website that detailed the specific benefits they would see because of the transition, including streamlined billing and making it easier to get patient records from other hospitals and providers. The website also shared some of the short-term "bumps in the road" patients and families could expect, such as longer wait times to see a healthcare provider due to short-term changes, as well as the need to reenter some patient demographic information and revisit medication lists.

The team was careful to not wallpaper the medical center with signage, based on feedback from the councils. They had confirmed that too many signs create unnecessary confusion and visual clutter. One patient noted that it would be "frankly alarming" if there were signs everywhere discussing the change.

What the carefully deployed signs did do is direct patients and families to speak to an employee for more information. The team recognized that a caring message from VUMC staff was going to be much more impactful than language read on a sign or a website. In fact, many employees wore campaign stickers during the transition to encourage conversations with patients regarding the change VUMC was experiencing. As such, workforce preparation was a critical aspect of the workgroup's efforts, as detailed in the previous section.

Changes to the billing system also required some direct communication, which were easily addressed through inserts in patient bills and statements leading up to and following the transition. More complex, though, was the third patient-visible variation identified by the workgroup: changes to VUMC's patient portal, My Health at Vanderbilt. More than 200,000 patient portal members had to take specific actions to transition their accounts to the new portal, which was now powered by Epic functionality.

Guided by the advice of the workgroup's patient and family representatives that cautioned VUMC to "only tell us what we need to do, when we need to do it," the team developed specific email and letter communications that minimized message volume and length and focused on clear, action-focused language. It was crucial that the entire 
campaign be very strategic in terms of timing, number of messages, and content that was incredibly precise with distinct calls to action (Figure 7).
Step 6: Track patient feedback during the change VUMC realized the importance of measuring the transition's impact on the patient experience during go live and therefore initiated an "in-the-moment" survey process that asked patients a few critical questions about their experience that day. Delivered to patients at various valet

Figure 7. MHAV Communications

\section{MHAV Communications}

\section{Key Points}

- The My Health at Vanderbilt (MHAV) web portal will be updated to a new version powered by Epic MyChart

- ALL MHAV web users must update their passwords: first login on/after November 2

- SOME users must verify additional account details: first login on/after November 2

- Mobile app users must update their app

\section{Communication Goals and Guideposts}

- Provide the necessary information for users at appropriate times (information in context)

- Simplify users' "work"

- Always offer an option to talk to support

Figure 8. Exit Survey at Valet

\section{Exit Survey at Valet}

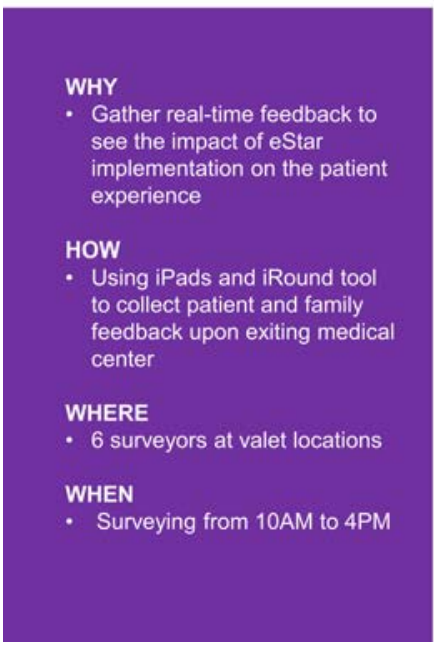

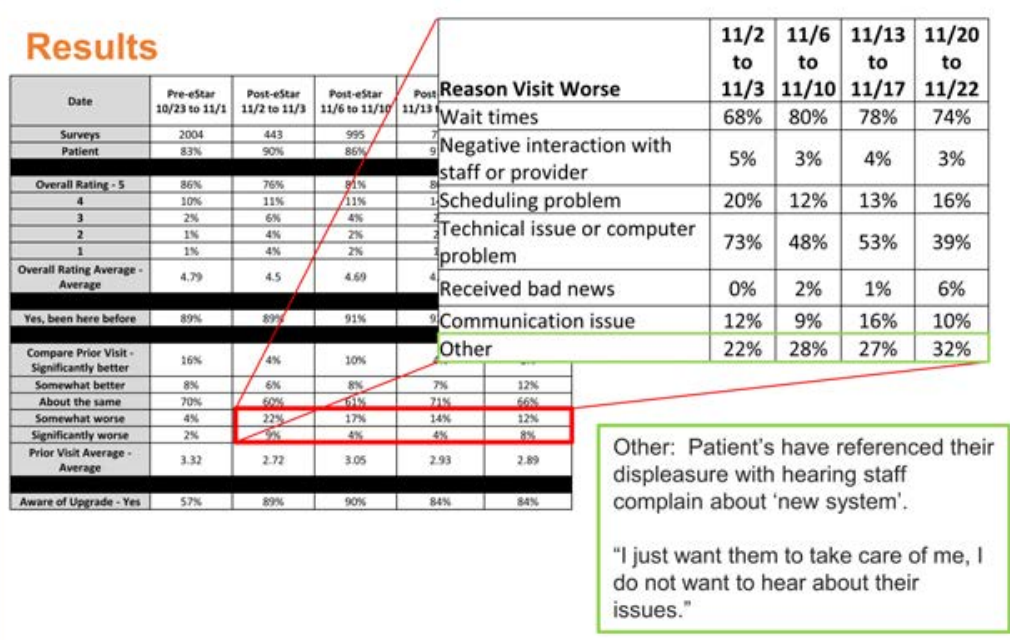


Figure 9. Patient Experience Scores - Pre/Post EMR Implementation (26 Systems Benchmarked)

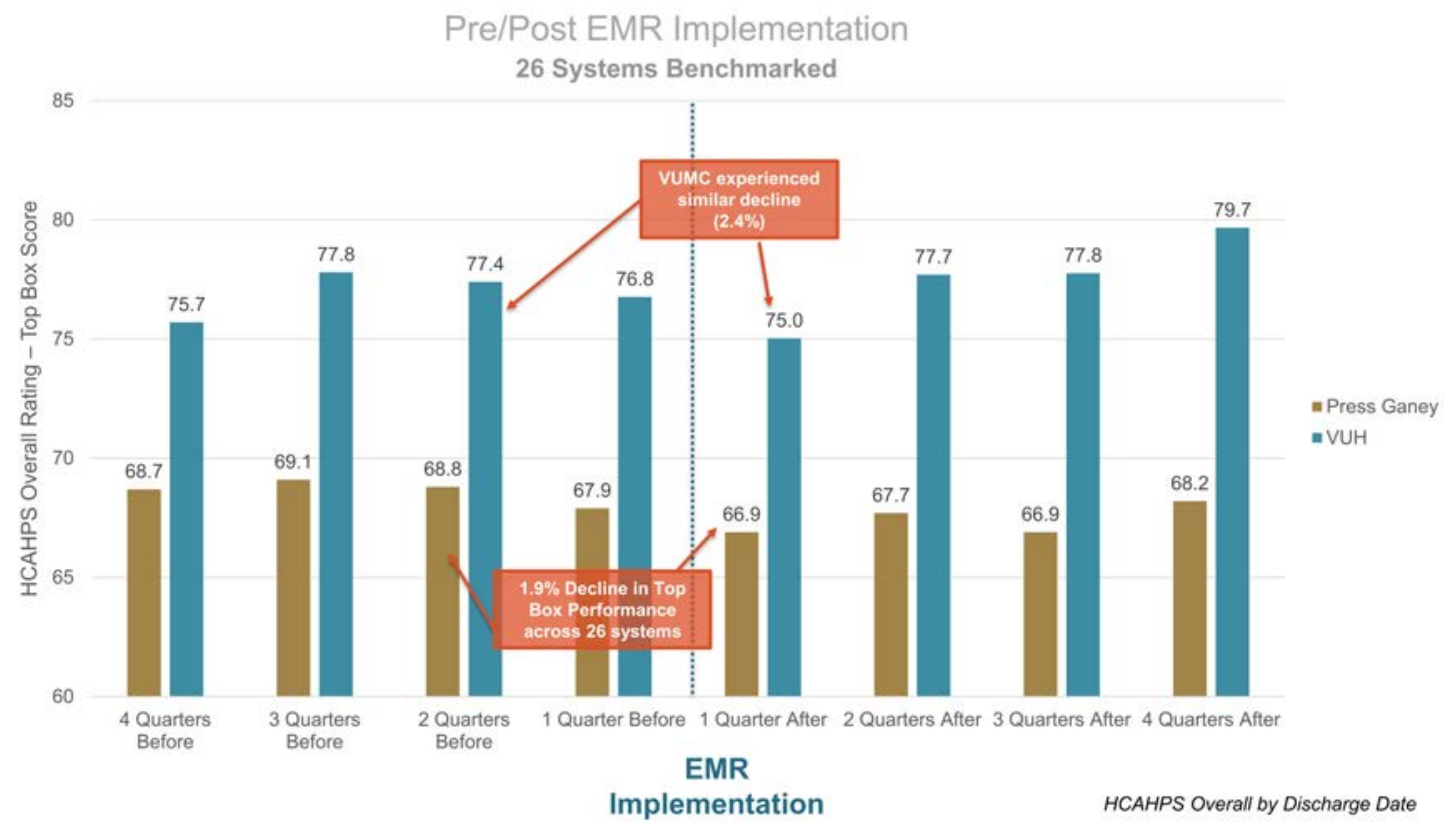

parking stations across the medical center and staffed by a handful of temporary workers, the optional survey captured patient feedback in an otherwise passive moment (as they were waiting for their cars). Using this innovative approach, VUMC gained a real-time pulse check of the patient experience during the transition (Figure 8).

\section{Step 7: Correct course as needed}

Throughout the weeks following the go live, VUMC executive and operational teams huddled several times a day to track and address a wide variety of issues. During these huddles, they reviewed data from the valet surveys to gain daily intelligence and insight into the current experience of VUMC patients. Survey data was used as a guide to identify where problems might be occurring, the best resources to further explore the issues, and optimal methods for resolving them as quickly as possible. Because of the daily touchpoints from the surveys, VUMC could see, almost in real time, if its mitigation efforts were having a tangible impact on the patient experience.

\section{Outcomes}

While the need for the daily valet-based data wore down in the weeks following go live, VUMC continued to use traditional survey methods to track patient experience scores. This survey data helped VUMC benchmark its EHR implementation's effects on the patient experience against other health systems. Unlike its peer institutions, which took an average of four quarters to see a return to pre-go live scores, VUMC was back to its pre- implementation scores by the second quarter following the transition (Figure 9).

Though it is difficult to pinpoint a single explanation for this success, a large part of that could be attributed to the strong efforts to prepare patients for and communicate with them regarding the transition. It is also likely that the awareness generated with the workforce helped them remember that the change or stress they were feeling was not the patient's fault or issue. Several employees commented after training that it was a good reminder for them to be aware of how they were presenting their stress or anxiety to the patient.

\section{Recommendations}

While VUMC's experience was specific to an EHR implementation, the seven-step strategy it used to prepare its patients for the effects of the transition should be beneficial for any organization facing a disruptive change. These steps include:

1. Gain the patient perspective. While not all organizations have formal advisory councils such as those in place at VUMC, there are other ways to gain insight into patients' views of how a change might affect them. Surveys and focus groups are ideas for helping organizations procure opinions and guidance from their patients. 
2. Get the support of leadership. The experience of VUMC demonstrates how important it is to have senior leadership on board with supporting patients through the downstream impacts of a large-scale change. Leaders can help prioritize, clear roadblocks and assign resources when needed. Leadership support can take the shape of a formal governance structure or be more ad hoc involvement when necessary.

3. Think through all the potential impacts patients and families might experience. Engaging in this formal thought exercise-which likely requires the involvement of several representatives from different areas of the organization-may result, as it did for VUMC, in a lengthy list of possibilities. Organizations can take this list and cull from it those impacts which they think merit the most time and energy.

4. Prepare your workforce to engage with patients. Organizational employees must be prepared with the right talking points regarding the change to ensure patients receive consistent messaging during the change. Disseminating and reinforcing this information with employees should reflect the culture and realities of each individual organization. For example, some organizations respond well to coaching in small team meetings with their front-line leaders, while others are more receptive to critical messaging that comes directly from senior leadership.

5. Communicate smartly and strategically with patients. When facing transformational change, some organizations err on the side of over-communication, which might overwhelm patients and create unnecessary alarm. Organizations that undercommunicate risk reputational issues (if patients think that the change was being covered up), or patient confusion or dissatisfaction. Organizations need to determine the right messages, channels and timing for how they are informing patients of the impacts of a large-scale change.

6. Measure effects of preparation efforts. Data is critical to understand the impact of an organization's patient preparation efforts. Organizations should establish a benchmark and goal for where they are prechange and where they want to be post-change, and then implement a measurement tool that allows them to track their efforts.

7. Use feedback to allocate resources and re-direct efforts. The value of data is not only that it gives insight into whether patient preparation efforts were successful, it also allows organizations visibility into specific areas or issues that need resolution. Organizations can determine whether they need to course-correct on any of their efforts, or direct resources to identified "pain points".

Depending on the change effort or project scope, an organization may not need to follow all seven steps. It is suggested, though, that no matter the project, the most essential element is Step 1. It is critical to any successful organizational change that customers/end users/patients be included in the planning process, as they can assist in identifying the most meaningful effects to their experience post-change.

\section{Looking Forward}

The healthcare sector has a reputation for being notoriously change-adverse, ${ }^{21,22,23,24}$ but a confluence of market forces, regulatory realities and consumer behavior $25,26,27$ is forcing healthcare organizations to become more adept at dealing with the realities of change. ${ }^{28,} 29$ As such, many organizations are borrowing "best practices" from outside the sector, better understanding how these industries execute thoughtful and effective change management. $30,31,32,33$

As a suggestion for future research in this area, it is worth considering how healthcare continues to evolve its approach to change management to align more with how the discipline is approached in other sectors, such as retail, finance, technology and transportation. The example of the retail industry is particularly interesting, ${ }^{34}$ given much discussion around the ongoing consumerization of healthcare. ${ }^{35}$ Additionally, the lack of specific research in this area stresses the important of future insights into how healthcare organizations consider and measure the patient experience during an organizational change.

\section{References}

1. Arndt R. CEO Power Panel: Health systems find consumerism drives innovation. Mod Healthc. https://www.modernhealthcare.com/article/20180519 /NEWS/180519911/ceo-power-panel-health-systemsfind-consumerism-drives-innovation. Published May 19, 2018. Accessed September 16, 2019.

2. Barrett AK. Electronic health record (EHR) organizational change: explaining resistance through profession, organizational experience, and EHR communication quality. Health Commun. Apr 2018;33(4):496-506.

3. Kash B, Spaulding A, Johnson C, Gamm L. Success factors for strategic change initiatives: a qualitative study of healthcare administrators' perspectives. J Healthc Manag. 2014;59(1):65-81.

4. Slosberg M, Nejati A, Evans J, et al. Transitioning to a new facility: the crucial role of employee engagement. J Healthc Manag. 2018;63(1):63-77. 
5. Valentino C. The role of middle managers in the transmission and integration of organizational culture. J Healthc Manag. 2004;49(6):393-404.

6. Le Tourneau B. Managing physician resistance to change. J Healthc Manag. 2004;49(5):286-288.

7. Weiner, BJ. A theory of organizational readiness for change. Implement Sci. 2009;4(1):67.

8. Aiken LH, Sochalski J, Lake E. Studying outcomes of organizational change in health services. Med Care. 1997;35(11):NS6-NS18.

9. Migdal C, Namavar A, Mosley V, Afsar-manesh N. Impact of electronic health records on the patient experience in a hospital setting. J Hosp Med. 2014;9(10):627-633.

10. Eiman A. Exploring patient satisfaction before and after electronic health record (EHR) implementation: the Kuwait experience. Perspect Health Inf Manag. 2013;10(Spring):1c.

11. Jarvis B, et al. Assessing the impact of electronic health records as an enabler of hospital quality and patient satisfaction. J Assoc Am Med Coll. 2013;88(10):14711477.

12. Liu J, Luo L, Zhang R, Huang T. Patient satisfaction with electronic medical/health record: a systematic review. Scand J Caring Sci. 2013;(27): 785-791.

13. Press Ganey Healthcare Metrics Team. HCAHPS Scores after EMR Conversion. Press Ganey Associates Inc. Published 2015.

14. Brown G. Managing your customers thorough change. Entrepreneur. https://www.entrepreneur.com/article/316161. Published July 3, 2018. Accessed October 7, 2019.

15. Merlo O, Eisingerich A, Auh S. Why customer participation matters. MITSloan Manage Rev. 2014;55(2):81-88.

16. Too EG, Weaver P. The management of project management: A conceptual framework for project governance. Int J Proj Manag. 2014:32(8):1382-1394.

17. Jones L, et al. Employee perceptions of organizational change: impact of hierarchical level. Leaders Organ Dev J. 2004;29(4):294-316.

18. Smith I. Achieving readiness for organisational change. Libr Manag. 2005: 26(6/7):408-412.

19. Vanderbilt Health Welcomes eStar [video online]. 2017.

https://www.youtube.com/watch?v=BqpPztOORgg. Accessed September 16, 2019.

20. Kristen 1. Carman, Pam Dardess, Maureen Maurer et al., Patient and Family Engagement: A Framework For Understanding The Elements And Developing Interventions and Policies. Health Affairs. Feb 2013; 32(2).

21. Appelbaum S, Wohl L. Transformation or change: some prescriptions for health care organizations. Managing Service Quality: An International Journal. 2000;10(5):279-298.
22. Dubois CA, Bentein K, Mansour JB, Gilbert F, Bédard JL. Why some employees adopt or resist reorganization of work practices in health care: associations between perceived loss of resources, burnout, and attitudes to change. Int J Environ Res Public Health. Dec 20 2013;11(1):187-201.

23. Narine L, Persaud, D. Gaining and maintaining commitment to large-scale change in healthcare organizations. Health Serv Manage Res. 2003;16(3):179-187.

24. Smythe, R. Why changing health care is hard. Forbes. https://www.forbes.com/sites/roysmythe/2014/02/2 4/why-changing-health-care-is-hard/\#1803ce124f1b. Published Feb 14, 2014. Accessed September 16, 2019.

25. Delmatoff J, Lazarus I. The most effective leadership style for the new landscape of healthcare. J Healthc Manag. 2014;59(4):245-249.

26. Knight S, Sorin D. A new healthcare alliance: consumer engagement in the new healthcare economy. Healthc Transf. 2016;1(3). DOI:10.1089/heat.2016.29015.skd.

27. Rutherford R. Five trends in healthcare that will change the way managers manage. J Med Pract Manage. 2017;32(4):239-242.

28. Laiteerapong N, Huang ES. The pace of change in medical practice and health policy: collision or coexistence? J Gen Intern Med. 2015;30(6):848-52.

29. Chatfield JS, Longenecker C, Fink L, Gold J. Ten CEO imperatives for healthcare transformation: lessons from top-performing academic medical centers. J Healthc Manag. 2017; 62(6):371-383.

30. Toussaint J, Berry L. The promise of Lean in healthcare. Mayo Clin Proc. 2013;88(1):74-82.

31. Chowdhury J. Hacking health: bottom-up innovation for healthcare. Tech Inn Man Rev. July 2012:31-35.

32. Black J. The Toyota Way to Healthcare Excellence: Increase Efficiency and Improve Quality with Lean. 2nd ed. Chicago: Health Administration Press; 2016.

33. Mazzocato P, Savage C, Brommels M, Aronsson H, Thor J. Lean thinking in healthcare: a realist review of the literature. Qual Saf Health Care. 2010;19(5):376-82.

34. Chopra P, Saunders N. Omni channel health: Envisioning Sarah's retail consumer experience in healthcare. Healthc Transf. 2015; 1(1). DOI: 10.1089/heat.2015.29005-pch.

35. Lineweaver N. The Healthcare Consumerization Report: How Healthcare Providers Can Transform Their Services to Meet the Evolving Needs of Digital Consumers Before, During and After Care. Business Insider Intelligence. Nov 2018. https://store.businessinsider.com/products/thehealthcare-consumerization-report 